\title{
Editorial
}

\section{Los tratados de libre comercio y las migraciones: Un interrogante}

Los tratados de libre comercio (TLC) y las migraciones son dos fenómenos mundiales, mútuamente relacionados, que nos plantean un interrogante: los tratados de libre comercio, ¿son la solución adecuada para frenar los crecientes flujos de migraciones mundiales? En la cumbre mundial sobre el Desarrollo Social (ONU, Copenhague, 1995) aparece la figura del emigrante. "Una de cada 115 personas del planeta es un emigrante o refugiado, que se ba visto obligado a abandonar su país por razones económicas, politicas o militares. Un millón de emigrantes se trasladan del Sur al Norte cada año. La cifra de refugiados políticos y victimas de conflictos étnicos aumentó de 8 millones a fines del decenio de 1970 a 20 millones. La migración misma ha pasado a ser un negocio a veces tan lucrativo como el tráfico de droga. Estas son algunas estadisticas que muestran que vivimos en un mundo enfermo". La cumbre de Copenhague, convocada por la ONU, presenta estadísticamente tres problemas mundiales: se desarrolla la pobreza, el crecimiento con desempleo y la atomización e insolidaridad social. Boutros Ghali dijo que la crisis de desempleo es, al mismo tiempo, una 
crisis económica, una crisis social, una crisis moral de incertidumbre y una crisis de gobernabilidad. ("Entorno económico mundial", UCA Editores 2003; pp. 85-91)

Desde este escenario mundial podemos contemplar los dos problemas nacionales: los 2,200,000 emigrantes salvadoreños en los Estados Unidos y los crecientes déficits de la balanza comercial, fenómenos mútuamente relacionados. Son más los empleos que generamos en el extranjero con la creciente demanda de importaciones, que los empleos nacionales generados por el monto menor de nuestras exportaciones. Una muestra aritmética es que en la revista del Banco Central de Reserva, el listado de nuestras exportaciones ocupa tres páginas y las importaciones, únicamente nueve. La diferencia aritmética se traduce en el creciente déficit de la balanza comercial, es decir, en un desempleo estructural. Hay una relación directa entre el flujo de las migraciones y la crisis interna de desempleo. Esto significa que 'el eje de las exportaciones' no ba funcionado en la década de los noventa, razón por la cual el presente gobierno pone toda su confianza (teoría o dogma) en la imparable secuencia de tratados de libre comercio, ya sea que éstos se firmen con economías similares o con el gigante de todas las economías. Se dirá que, dada la estrechez del mercado interno, las empresas ampliarán sus escalas de producción, con mayor empleo, si entran a competir en el océano abierto del mercado mundial.

Para ello, se bacen necesarias largas negociaciones y tratados de "libre comercio", calificativo bastante cuestionado en las cumbres de la Organización Mundial del Comercio (OMC), desde Seattle a Qatar y más allá de Qatar hasta Cancún. Los tratados de libre comercio significan, históricamente, que las economias menores tratan que el comercio mundial sea más equitativo y más libre. Los expertos en la materia distinguen los tratados de libre comercio entre economías similares, de los tratados entre economías desiguales, dando a entender que difiere ampliamente "el grado de libertad" en ambos escenarios. Abi radica el problema, y es lógico que crezca el debate - a escala mundial y nacional - sobre los esperados beneficios económicos y sociales del comercio internacional en-

\section{6}


tre desiguales, porque también las negociaciones serán desiguales. Recordemos que el comercio internacional es uno de los ejes donde se gesta "el malestar en la globalización", como le ha dado en llamar el economista Joseph Stiglitz.

He aqui unos pocos titulares. La revista de Comercio Internacional, publicada en México, dedica su número de diciembre de 2002, a "El sinuoso camino del comercio mundial", desde Seattle hasta Doha, Qatar. Por su parte, FUSADES dedica el número 3 de sus investigaciones del año 2002 a "Las prácticas desleales del comercio internacional y su relevancia para El Salvador". FUN$D E$, que viene trabajando este tema en repetidas publicaciones, dedica el Tercer Avance, julio 2003, a la "Propuesta regional laboral y agricola ante la negociación del TLC entre Estados Unidos y Centroamérica", planteando, junto con la CID, una moratoria a las negociaciones de este TLC. El Centro de Información sobre Inversión y el Comercio Centroamericano (CEICOM) presenta su estudio sobre los "Impactos en El Salvador del Tratado de Libre Comercio con México", abril 2003. Estas y otras instituciones analizan nuestros TLC desde el escenario de la tupida red de tratados de libre comercio mundiales, es decir, desde el escenario de la globalización. El tema álgido es el funcionamiento de la globalización, uno de cuyos ejes centrales es el comercio internacional. Se plantea el interrogante: ¿aliviarán los TLC los flujos de migraciones y el flagelo del desempleo?

\section{Los tratados de libre comercio, ¿̇una "moda" o un "modelo"?}

El mapa de la geografía económica mundial se parece a los mapas de las compañias de aviación, donde nos muestran la extendida red de conexiones intra e intercontinentales. Es una imagen cercana de la estrecha red de tratados comerciales. Estados Unidos firma un TLC con Canadá y México, países vecinos, y acaba de firmar otro con Chile, país más lejano. Canadá y el Salvador firman otro TLC, al mismo tiempo que Vicente Fox, mandatario de México, aprovecha la cumbre de Davos 2001, en Suiza, para firmar un acuerdo similar con la Unión Europea, y también ha firmado otro 
con el triángulo Norte de Centroamérica -Guatemala, Honduras y El Salvador- Hemos firmado un TLC con República Dominicana $y$, junto con el resto de paises del istmo, se llevan a cabo largas y no muy transparentes negociaciones para la apresurada firma del TLC con los Estados Unidos, como un paso previo a la ratificación del Área de Libre Comercio de las Américas (ALCA) en el 2005. La más reciente propuesta es un acuerdo de libre comercio con Taiwan.

No son éstas las únicas interconexiones mundiales. Los 'tigres asiáticos', sacudidos por la crisis financiera mundial, 1997-98, vuelven a formar un bloque poderoso en el continente sudasiático. China se integra a la OMC en 2001 y nuevos paises del Este europeo se integran o hacen fila para adherirse a la zona euro. También el Próximo Oriente ha sido invitado a unirse a un bloque de acuerdos de libre cambio. El Mercosur latinoamericano mantiene sus antiguas relaciones comerciales con Europa, al mismo tiempo que participó en la cumbre de Quebec, abril 2001, invitado a adberirse al Area de Libre Comercio de las Américas, 2005.

Para esclarecer las primeras o segundas intenciones que entretejen estas tupidas redes comerciales, hay que hacer referencia a dos recientes propuestas de TLC o acercamientos comerciales. El pasado mes de junio 2003 se reúnen en Jordania un millar de miembros del foro económico de Davos, de representantes del "Cuarteto para el Oriente Próximo" (EEUU, UE, Rusia y la ONU) y representantes de países árabes. El objetivo del foro económico es impulsar el diálogo y la reconciliación en Oriente Próximo. Los representantes de EEUU, Collin Powell y Robert Zoellick, aprovechan la oportunidad para presentar una "boja de ruta económica", con el objetivo de crear "una zona de libre cambio" con el Oriente Próximo de aqui al año 2013, que sirva a sostener los acuerdos de paz en la zona: "Nosotros queremos la paz en la región, pero es necesario que la paz se acompañe de un desarrollo económico, a beneficio de los pueblos de la región". Zoellick explicó que la hoja de ruta económica del libre cambio entre Estados Unidos y la región se irá estableciendo "paso a paso", en un proce- 
so que absorberá a los estados que firmen acuerdos bilaterales de libre cambio con Washington y que englobe un acuerdo regional con paises del Próximo Oriente y Norte de Africa. Estados Unidos ayudará con mil millones de dólares para iniciar las negociaciones con diversas agencias gubernamentales. Los paises invitados son Jordania (que ya tiene un TLC con EEUU), Babrein, Egipto, Marruecos, Omán, Qatar, Arabia Saudita, Túnez. Emiratos Arabes, Yemen, Liga Arabe y la Autoridad Palestina. No ban sido invitados Siria, Libia ni Sudán. (Ibídem; pp. 281-282)

Aqui se esconde algo más que libre comercio, pero también hay comercio. En el mes de julio 2003, la comitiva estadounidense, presidida por George W. Bush, hace una gira por paises de Africa occidental, en razón de lo que denominan "la década de África". El Golfo de Guinea va a ser el sustituto del Golfo Pérsico. Este golfo lo componen una serie de paises ricos en yacimientos petroliferos: Nigeria, Guinea Ecuatorial, Camerún, Gabón y Angola. Las enormes reservas petroliferas de estos países y la calidad del crudo aseguran una independencia frente a las oscilaciones de precios $y$ cantidades de la OPEP, con la ventaja adicional de que la extracción se realiza en la plataforma marítima, algo alejado de las perturbaciones sociales que puedan generarse en el interior de esos países. (El País, 12. 07. 03)

Ante esta estrecha red de TLC, no podemos bablar de una 'moda', sino de "un modelo" que se llama globalización y, más en concreto, los principios del Consenso de Washington, del cual extraemos los dos primeros mandamientos: "la subordinación del papel del Estado al del mercado y la máxima participación posible en los intercambios internacionales y la promoción del comercio exterior". Estos principios aparecen en la publicación de la ANEP, con motivo del II Encuentro Nacional de la Empresa Privada, ENADE 2001: "El actual modelo económico del pais se fundamenta en los principios filosófico-doctrinarios de la economía de mercado. Las bases de este modelo fueron trazadas hace más de una década (FUSADES) y con el tiempo ban tendido a estructurarse a través de un proceso de desregulación, privatización y eliminación de con- 
troles de parte del Estado, apoyados en un proceso de apertura frente al exterior". (ECA, 2003; pp. 545 y 543).

FUSADES confirma su fe en los frutos que generarán nuestros tratados de libre comercio. "Los salvadoreños ban llevado adelante reformas económicas y sociales que posicionan al pais relativamente bien para aprovechar los impulsos del crecimiento internacional, particularmente en el futuro cercano, cuando maduren las oportunidades de los tratados de libre comercio ya firmados, y cuando se abran las nuevas oportunidades que traerá la próxima firma del tratado de libre comercio con Estados Unidos". ("Competitividad para el desarrollo", agosto de 2003; p.1) Claramente vemos que no se trata de un "modelo" nacional (FUSADES, ARENA, ANEP), sino de un "uniforme" mundial, impuesto por el Consenso de Wasbington y asociados $y$, entre ellos, el FMI, como lo explica Stiglitz en su obra El malestar en la globalización. Los modelos económicos son una 'religión', con su credo, sus dogmas, su inquisición y sus adeptos, cuya característica histórica ba sido negarse a la autocrítica. La fe y la credibilidad de un modelo económico se confirman al enfrentarlo con la historia. Toda esa serie de tratados de libre comercio y sus mismas 'negociaciones' están supeditados a los "normas vigentes", que se han impuesto en las cumbres de la Organización Mundial del Comercio y es, a esta luz, que podremos deducir si tendrán un impacto positivo en la contención de los flujos de migraciones.

Una muestra representativa: Las cumbres de la Organización Mundial del Comercio (OMC)

Quien baya seguido la historia de las cumbre de la OMC, desde Seattle a Qatar y después de Qatar hasta Cancún, se dará cuenta de que la teoría del libre comercio no es, en la bistoria real, un comercio libre donde jueguen con transparencia las simples ventajas comparativas. En la cumbre de Seattle, EEUU, celebrada en diciembre de 1999, estalla 'el malestar en la globalización'. Unos 50.000 manifestantes dicen estar en la calle "para dar la palabra a aquellos que jamás la ban tenido y para que la OMC escuche la voz de los ciuda- 
danos. La ley de las multinacionales no es la democracia. La OMC es la Babilonia del segundo milenio, una especie de siniestra organización de rostro anónimo, convertida en el motor de una globalización que sólo favorece a las grandes multinacionales". Seattle es una partida de ajedrez que se juega desde los cuatro lados. Estados Unidos y algunos asociados contra Europa y Japón, con las figuras blancas y negras, y ambos a dos contra los paises pobres y emergentes, que sólo cuentan con un peón, que será "el peón de la victoria".

El borrador de un pacto a firmar contenía los siguientes temas. Sustanciales reducciones de las subvenciones agricolas europeas, la creación de un grupo de trabajo sobre biotecnologia, la ampliación hasta el 2001 de la moratoria sobre tasas de comercio en Internet, incremento de los intercambios en finanzas y telecomunicaciones $y$ reformas de las leyes norteamericanas contra el dumping o economia desleal. Los delegados de los países pobres y emergentes desconocian el borrador porque no habian participado en su redacción ni tampoco participan en su discusión. Quedó claro que los europeos subvencionaban "su agricultura multifuncional" con $\$ 45.000$ millones y Estados Unidos con $\$ 22.000$ millones, además de defender la comercialización de alimentos transgénicos.

Un detalle interesante, que vuelve a aparecer en la OMC de Qatar y que en forma muy acertada lo trata FUNDE en las citadas publicaciones del 2003, es que el anfitrión, Bill Clinton, pusiera sobre el tapete de la discusión las "normas laborales". En su discurso solicitó "derechos laborales mínimos para todos los países del Tercer Mundo, [pues] no se trata sólo de bajar las barreras comerciales, sino de subir los niveles de vida". Esta intervención bumanista del ex gobernante nortemericano dio cauce a la ironia de los europeos: se trataba de una evidente contradicción entre un Clinton, convertido en defensor de los trabajadores, y unos Estados Unidos que no han ratificado tratados de la Organización Internacional del Trabajo (OIT). Para los paises en desarrollo, la sugerencia de Clinton generó el temor de que los países ricos impongan sanciones $y$ dificulten las exportaciones de los paises que no apliquen normas laborales. En las negociaciones de nuestro TLC, 
Regina Vargo, representante de los EEUU, afirma que uno de los requisitos del Congreso es el cumplimiento de las normas laborales $y$ medioambientales. Al unisono con FUNDE, estamos totalmente de acuerdo con la aplicación de estas normas; pero es una gran "bipocresia" que imponga estas normas un Congreso que no ba ratificado tratados de la OIT y que se ha negado a ratificar el protocolo de Kioto. Joseph Stiglitz utiliza muchas veces al calificativo de "bipocresia".

En las cumbres de la OMC el documento final debe ser firmado por unanimidad de los miembros; los delegados de los paises pobres y emergentes utilizaron "el único peón" que les habian dejado: se negaron a firmar el documento final. "En Seattle triunfó el desacuerdo de los emergentes y de los países en desarrollo, que se negaron a firmar acuerdos finales cuando ni siquiera habian sido consultados y atendidos en la redacción de los propios borradores. La voz de los sin voz fue su desacuerdo, que ba sido interpretado como una victoria. Los paises en desarrollo no tuvieron que firmar un documento, contra su deseo, porque no babian sido consultados. Los países africanos denuncian acremente su marginación en todo el proceso de negociaciones. No bay transparencia alguna en este proceso y aseveran que "americanos y europeos juegan con nosotros con la política del palo y la zanaboria". Cuando las discusiones, a puerta cerrada, se eternizaban y no filtraba la menor información, los delegados de los paises pobres aguardaban pacientemente en los pasillos. "No sabemos qué decisiones se van a tomar y una vez más se nos pedirá suscribir un texto que ni tendremos tiempo de leer". La OMC ha sido por fin obligada a entrar en razón. (Entorno económico mundial; pp. 151-156)

¿Han entrado todos en razón? En el año 2000 no pudo convocarse la cumbre de la OMC, pero el fantasma de Seattle se bizo presente en las cumbres de Davos 2000 ("La nueva economia"), del FMI y BM en Washington y Praga y en la cumbre del Milenio, ONU, Nueva York. Luego de redactar su obra, "El malestar en la globalización", J. Stigitz dirige una carta a los miembros del G-7 reunidos en Génova, julio 2001. "El sistema de comercio global 
está con problemas. Se predica el libre comercio como el evangelio en todas partes, pero parece que los paises no bacen caso de su propio mensaje, sus mercados permanecen cerrados a muchos de los productos de los países en desarrollo; subsidian a sus agriculturas en forma masiva, lo que bace imposible que los paises en desarrollo puedan competir. El mensaje del G-7 parece ser: bagan lo que decimos, no lo que hacemos" (Ibídem; p. 220)

Se esperaba, con cierto nerviosismo, la convocatoria de la OMC en Doha, Qatar, en noviembre de 2001, porque -inesperadamente- la fecha no era la más afortunad: a a dos meses de los ataques a las torres gemelas de Nueva York. El "terrorismo" pasaba a ser el problema "número uno" del mundo y los problemas del comercio internacional podian estancarse en una vía muerta. No bubo propiamente un documento final cerrado, sino una serie de compromisos de seguir discutiendo los mismos problemas en la sede de la $O M C$ en Ginebra. Un compromiso de que se seguirian reduciendo las subvenciones agricolas, pero reducción no significaba anulación, como volverá a aparecer en la OMC de Cancún. Hubo avances coyunturales en el sensible tema los "medicamentos" capaces de aliviar las pandemias del SIDA, malaria, tuberculosis, donde "el derecho de propiedad intelectual" (GATT) forcejea con el derecho de las naciones a asegurar la salud y vida de sus pueblos. En Cancún se ha logrado una media victoria, criticada por varias ONG. (Le Monde, 31.08.03)

Los Estados Unidos volvieron a sacar el tema de las "normas laborales", que se envió a un diálogo de la OMC con la OIT. Los europeos quisieron integrar el tema de las "normas medioambientales", flanco débil de los Estados Unidos y también las normas ambientales quedaron en la via muerta. Estadounidenses y europeos insistieron en los temas de las "inversiones y competencia", que el resto de paises se negó a tratar en esta cumbre, porque en forma disimulada se pretende introducir (todavia boy) las normas del Acuerdo Multilateral de Inversiones (AMI), con igualdad de trato $y$ condiciones para la inversión extranjera y nacional. La firmeza de la India obligó a que se prolongara de un día 
la cumbre, solicitando que Estados Unidos y Canadá redujeran los aranceles del textil de $23 \%$ a $3 \%$, so pena de no firmar el documento final. En resumen una reunión para la firma de muchos compromisos. (Ibídem; pp. 228-232)

Después de Qatar sigue Qatar. Los Estados Unidos siguen siendo criticados por las masivas subvenciones agricolas a sus granjeros (\$22.000 millones en 2002), que J. Stiglitz llama bipocresia: "Es la perfecta ilustración de la bipocresia de la administración Bush sobre la liberalización del comercio". Luego de cinco años de disputas y a solicitud de la Unión Europea "la OMC autoriza sanciones a Estados Unidos por \$ 4.000 millones". La OMC declara ilegal el sistema conocido como "Corporaciones de Ventas al Exterior", que permite a grandes empresas aplicar subsidios a la exportación mediante la utilización de paraísos fiscales, desde los cuales realizan negocios con el exterior, reduciendo basta un 30\% la carga impositiva. Asi han estado exportando empresas aeronáuticas y otras empresas industriales: Kodak, Microsoft, General Motors, Caterpillar, Chrysler y Boeing, por ejemplo. Una gran parte de cereales $y$ de soya salen por este canal. (Ibídem; $p, 255)$. Recientemente, la OMC, a petición de Europa y otros siete paises, ba pedido a los Estados Unidos que retire la sobretasa de 8 a $30 \%$ impuesta a productos siderúrgicos y al acero, por espacio de tres años. El informe de la OMC consta de 900 páginas, pero Estados Unidos todavia se resiste a aceptar la orden de la OMC. (Le Monde, 12,07,03).

En este escenario y ambiente se están llevando a cabo las negociaciones de 'los tratados de libre comercio'. El comercio internacional viene a solucionar una debilidad de todos los paises: no bay nación autárquica que pueda, con sus propios recursos, responder a todas sus demandas de consumo e inversión. En el escenario mundial quienes más necesitan de un libre comercio son los paises más pobres que reclaman un cauce abierto a sus exportaciones, en aquellos rubros en que cuentan con ventajas comparativas, es decir, donde la naturaleza les ha hecho 'competentes', para avanzar hacia otros niveles de competencia y de empleo. Los paises en desarrollo no quieren permanecer estáticos, monoexportadores, y quieren su- 
bir al tren de la globalización. En teoría y en principio todos estamos a favor del comercio internacional, pero lo contradictorio de nuestro comercio mundial es que "la globalización hace difícil la globalización".

La geografía mundial no es equitativa: el hemisferio Norte está tachonado de "mercados comunes" (aunque uno de ellos se desintegró) y han logrado 'ser competentes para ser competitivos'. El historiador Lester Thurow escribe en 1992 su obra Head to head: "La guerra del siglo XXI. La batalla económica que se avecina entre Japón, Europa y Estados Unidos". Ha pasado una década y las cumbres de la OMC nos muestran que las tres potencias ban becho del comercio internacional una batalla económica entre el Norte y el Sur. Han convertido el libre comercio internacional en "un fraude intelectual" (J. Stiglitz); piden a los paises en desarrollo que sean 'competitivos', pero, con las normas del comercio internacional, no les dejan ser 'competentes' y no les es posible contener los flujos de migraciones. Franz Hinkelammert lo ba dicho en forma gráfica: el mundo es un todo global, pero la globalización es parcial; es más lo que excluye que lo que integra.

\section{Nuestros tratados de libre comercio}

Era necesario contemplar el escenario mundial antes de descender a comparar la teoría con nuestra bistoria real. Es un becho confirmado que el eje de las exportaciones no ba funcionado en la década de los noventa y debemos admitir que parte del fracaso tiene raices internas, incluido el autocalificado sector productivo. El economista del Banco Central de Reserva, Salvador Osvaldo Bran, lo decía en 1997: "Un aspecto de singular importancia es cómo superar el patrón de comportamiento tradicional del sector empresarial que cree en el principio del menor esfuerzo tecnológico. El proteccionismo excesivo de varios decenios provocó que éste eludiera correr riesgos, en particular, los asociados a la innovación tecnológica. Muchos empresarios se ban acostumbrado a no competir con mejor calidad y precio; prefieren bacerlo con publicidad, acceso a crédito preferencial y la obtención de subsidios del gobier- 
no, es decir, se babituaron a formas de competencia que exigen poco o ningún esfuerzo tecnológico, práctica que debe erradicarse para ser más competitivos". (ECA, 1997; p. 546)

Cuatro años más tarde, la propia ANEP reconoce este fallo, al comentar el catalizador de la educación: "Un mundo moderno está basado en el conocimiento y los países que mejor produzcan y tengan acceso al mismo lograrán ventajas considerables. El Salvador presenta serias limitaciones para enfrentar los retos de la globalización y la revolución mundial del conocimiento, ya que el desarrollo de la ciencia y la tecnología en el país ha sido bistóricamente escaso y no se observan signos de mejora sustancial. Cambiar esta realidad exige un esfuerzo nacional. El sistema educativo juega un rol de primer orden en este proceso y la educación superior un papel estratégico en el corto plazo. La empresa y la academia deben integrarse en un ambiente de ganar-ganar" (ENADE 2001, p. 14) Estas reflexiones están a la base de "las diez propuestas para la competitividad", presentadas por FUSADES. Estas recomendaciones, que son obvias, ojalá bubieran sido publicadas en 1989, junto con el informe "Hacia una economía de mercado", o en 1992 cuando se instituye la Comisión de Ciencia y Tecnología.

Aunque tarde, es el momento de una reflexión que no se bizo en esos años. En la década de los ochenta, bajo diversas apariencias, el modelo que jugó fue "una economía de guerra" y "una guerra a la economía" desde ambos flancos. Lo que necesitaba nuestra desvertebrada economía en 1989 y 1992 era un "modelo de reconstrucción" física y moral. La teoría recomendó apoyarse en el trípode de la propiedad privada, el mercado y la competencia, con el apéndice de un Estado subsidiario. Esa fue nuestra "boja de ruta" que nos ha conducido a la vía muerta del estancamiento. Luego de doce años de fallida experiencia, nuestros gobiernos $y$ asociados vuelven a poner su total o mayor confianza en el libre comercio, con la peculiaridad de que la confianza es tanto mayor cuanto mayor sea la potencia de quien negocia del otro lado del TLC. La teoría es más fuerte que la bistoria. Volvemos a repetir que estamos 
a favor del libre comercio, justo y equitativo, lo cual suele darse más bien entre iguales.

\section{El tratado de libre comercio con México}

Con una economia que se venía debilitando desde 1996 y en compañia de Guatemala y Honduras, luego de largas negociaciones, firmamos en junio 2000 el TLC con México, una economía más sólida que la nuestra y más entrenada en el difícil arte de las negociaciones con Estados Unidos y Canadá. El Centro de Información sobre Inversión y Comercio en Centroamérica (CEICOM) publica un trabajo titulado "Impactos en El Salvador del Tratado de Libre Comercio con México", en abril de 2003. La investigación se circunscribe al impacto en las variables: comercio (exportaciones e importaciones), producción, salario, ganancia empresarial, ingresos fiscales, consumo intermedio y empleo. CEICOM promete llevar adelante esta investigación en el tiempo y otras variables. ¿Qué nos enseña este primer tratado de libre comercio?

Nuestro gobierno babia puesto grandes esperanzas y desmedida publicidad en este tratado, de manera especial el ministro de Economia, Miguel Lacayo: "Estamos hablando de una cantidad importantísima de empleo, con lo cual nuestro problema se termina. Todo el que quiera trabajar va a tener trabajo; la gente va a tener mejores sueldos; vamos a vivir todos una vida digna". El tratado generaría 15.000 empleos. (p. 54). No son éstas las conclusiones que emanan de la presente investigación, luego de dos años de firmado el tratado. "I) El comercio de El Salvador con México sigue siendo poco significativo en términos macroeconómicos, principalmente en lo relacionado con las exportaciones, las cuales, aunque casi se duplicaron en el primer año del TLC, sólo representan un $1 \%$ del total nacional. Además, en el segundo año las exportaciones redujeron sustancialmente su dinamismo... 2) La falta de competitividad de las empresas salvadoreñas en relación a la oferta exportable concentra los beneficios del TLC en pocas empresas, dejando fuera a los medianos y pequeños negocios. 3) Las importaciones de bienes mexicanos aumentaron bastante en el primer año y provocaron un mayor 
aa ae inversiones extranjeras para aprovechar la mano de obr barata y las legislaciones favorables al capital. Sin embargo, no ha. datos gubernamentales que demuestren que ello esté ocurriendo".

"5) Las variables de producción, empleo, ingresos (salarios, ga nancias e impuestos indirectos) y consumo intermedio, a pesar d que crecieron en el primer año del tratado, no alcanzaron nivele significativos y su impacto en la economía del pais fue casi imper ceptible. Además, en el segundo año casi llegaron al estancamientc 6) Del incremento de ingresos se benefició más, aunque ligeramen te, la ganancia que los salarios. El TLC, por lo tanto, no propici. condiciones para redistribuir el ingreso, que, junto al desempleo, $\epsilon$ la causa del alto indice de pobreza del país. 7) El empleo cread. por el crecimiento de las exportaciones es poco significativo y con centrado en algunas ramas productivas. En el segundo año del tra tado el empleo prácticamente se estancó y en varias ramas se redu jo. Los datos de los dos primeros años del tratado indican que $l$. meta del gobierno está muy lejos de ser alcanzada”. (Pp. 56-58)

"En realidad sucedió lo contrario: la economía salvadoreña viv boy de importaciones financiadas con remesas. Tanto el sistem. financiero como el resto de sectores dependen de la evolución d las remesas, sin las cuales no sería posible mantener el flujo $d$ importaciones que demandan el aparato productivo y el sector $d$ servicios. Alrededor del comercio importador y del sistema finan ciero se aglutina la nueva cúpula empresarial surgida del fin de l. guerra y desarrollada por la política económica de los últimos tre gobiernos". (p. 14) Aunque estamos en los dos primeros años d. experiencia, no podemos deducir que los tratados de libre comerci

\section{8}

Reolidad 95, 200 
van a tener un impacto sensible en la reducción de las migraciones, cuando son las migraciones, traducidas en remesas, las que bacen posible sostener nuestro comercio externo. Esto significa que existen algunos interruptores o cortacircuitos internos que impiden la aparición de los resultados esperados.

En este sentido es cierto el análisis de FUSADES: "Competitividad para el Desarrollo". Pero si el modelo recomendado en 1989 era la economía de mercado, basado en la competencia, ¿por qué los gobiernos de turno no aplicaron estas recomendaciones, a partir de 1992, luego de los acuerdos de paz? Y, ¿̇ómo se podía ser competitivo con una economía desvertebrada (terciarización económica), simplemente dirigida por programas monetarios y financieros? Se archivaron las matrices insumo-producto 1978 y 1990, que nos mostraban qué sectores podian dinamizar la economía, si a ellos se dirigiera el crédito bancario. Tenemos demasiadas universidades para la formación de 'cuellos blancos'y muy pocas escuelas profesionales para la capacitación de mano de obra calificada. Se descuidó estructurar el sector real, la capacitación de mano de obra y el crédito bancario corrió por cauces improductivos; abora tendremos que esperar al año 2015 ó 2025 para poder ser competitivos. Falló el modelo de economía de mercado precisamente cuando se estaba haciendo más agresivo y competitivo el mercado internacional.

\section{Las prácticas desleales del comercio internacional}

Carlos Orellana Merlos (FUSADES) inicia esta investigación con una frase orientadora: "Si bien es cierto que, en general, la apertura comercial externa resulta ventajosa para los consumidores y que puede mejorar la eficiencia de la economia, creando un esquema económico más competitivo, también puede generar situaciones de peligro para la producción nacional cuando aparecen en el mercado lo que se conoce como 'prácticas desleales del comercio internacional" ( $p .1)$. Se trata de un análisis aplicado, que explica los términos conceptuales de las prácticas desleales $y$, a partir de una encuesta pasada a 475 empresas de distintos sectores y tama- 
tantes empresas afirman haber sentido los impactos-dumping desde 1998, cuyos efectos mayores ban sido la reducción del margen de ganancia, la caida de los precios y una sensible pérdida del mercado interno por dumping $y / o$ subsidios externos. "¿Qué paises considera que ban aplicado este tipo de subsidios en sus procesos de producción o comercialización?" ¿Quiénes practican el dumping? El listado es interesante: "Estados Unidos, Unión Europea, Australia y Japón, China, Rusia, Singapur, Vietnam, Taiwan, México, Brasil, Colombia". Este listado confirma lo que claramente aparecía en las cumbres de la OMC. No todos han entrado en razón.

Son pocas las empresas que ban denunciado estas prácticas desleales en el mercado interno y externo, en razón de la complejidad de estos procesos, los costos del monitoreo, las ineficientes y burocráticas oficinas públicas, la falta de personal calificado en el sector gubernamental y el marco jurídico e institucional inadecuado. Es poco significativo el aprecio por los servicios que presta el gobierno para contrarrestar estos efectos y la mayoría de empresas "considera que el Gobierno no ba becho nada; un grupo bastante numeroso de empresas están exigiendo implícitamente al Gobierno un cambio en su actitud vinculada con las prácticas desleales del comercio internacional". Un $63 \%$ de las empresas piensa que no mejorará o se incrementará esta práctica de políticas desleales en los países competidores. (pp. 37-48)

Esta encuesta ilumina bastante el hecho de que el libre comercio internacional, no sólo pone serios obstáculos al ingreso de nuestras exportaciones, sino que además está reduciendo el campo del mer- 
cado interno a nuestras empresas. Hoy dia se agregan otras barreras desleales que está imponiendo el gobierno de Estados Unidos: las medidas sanitarias y fitosanitarias, a las que se agrega la nueva normativa "en materia de bioterrorismo". Estos obstáculos son analizados por FUNDE en su Tercer Avance: "Propuesta regional laboral y agrícola ante la negociación del TLC entre los Estados Unidos y Centroamérica”, publicada el mes de julio de 2003.

"El acceso al mercado de los Estados Unidos exige el cumplimiento de normas sanitarias rigurosas (etiquetado, aditivos, residuos de plaguicidas, control de procesos, entre otros), principalmente en productos para el consumo bumano. En la práctica dicho país ha convertido las MSF en barreras que restringen el ingreso de la producción de Centroamérica hacia su mercado. Los Estados Unidos exigen para el reconocimiento de equivalencia del estatus sanitario de los países el cumplimiento de rigurosas normativas elaboradas y administradas por ellos mismos, dándoles un amplio margen de discrecionalidad en cuanto al cumplimiento de plazos, inspecciones y finalmente en las autorizaciones. En reiteradas ocasiones estas normativas constituyen barreras técnicas al comercio. De igual manera, debe prestarse particular atención a la nueva normativa en materia de bioterrorismo de parte de EEUU, con el fin de evitar que esta legislación se constituya en una barrera técnica al comercio, que introduzca nuevos requisitos que de forma injustificada traten de encarecer y/o complicar los procesos de exportación de Centroamérica". (p. 36)

En virtud de la defensa del libre comercio, deducimos que ciertos tratados de libre comercio no debieran iniciarse ni firmarse. Esa fue la respuesta de Seattle, que se interpretó como una victoria. Algo anda muy mal cuando, del 8 al 10 de agosto, más de 200.000 manifestantes se reúnen en Larzac, Francia, para contraatacar la próxima cumbre de la OMC en Cancún: "Es necesario someter a nuestros gobiernos bajo el control de los ciudadanos". Debemos combatir la "regresión social" y luchar para que "la vida triunfe sobre la OMC”. (Le Monde, 12.08.03). 